Alexander Olk · Friedrich Frank Hennig

Abteilung für Unfallchirurgie, Chirurgische Klinik mit Poliklinik, Universität Erlangen-Nürnberg

\title{
Navigation und Robotronik in der Knieendoprothetik
}

\section{Aktueller Stand}

\section{Zusammenfassung}

Der breite Einsatz von Robotern und Elektronik in der Fertigung nahezu sämtlicher Gegenstände des Alltagslebens legt die Frage nahe, ob diese Technologien nicht auch im Rahmen operativer Eingriffe zum Nutzen der Patienten eingesetzt werden können. Dieser Einsatz sollte nicht als Konkurrenz für den Operateur, sondern eng begrenzt auf genau beschriebene Operationsabschnitte, im Sinn eines Helfers, in der Hand eines erfahrenen Operateurs gesehen werden. Nach erfolgreicher Einführung der roboterassistierten und navigierten Operation Mitte der 1990er Jahre werden diese Verfahren nun zunehmend auch in der Knieendoprothetik eingesetzt.

\section{Schlüsselwörter}

Robotik · Navigation · Endoprothetik . Oberflächenersatz $\cdot$ Knie lloarthroplastische Eingriffe an den großen Gelenken lassen sich in mehrere große Abschnitte unterteilen. Eine umfangreiche präoperative Planung hat das Ziel einer trajektorengerechten Implantation der Prothese, häufig unter Korrektur vorbestehender krankhafter Zustände der Achsverhältnisse (Abb. 1) und unter besonderer Berücksichtigung des Weichteilmantels. Letztendlich besteht die Zielsetzung, Muskelfunktion und Kapselspannung ausgewogen der durch das künstliche Gelenk geschaffenen neuen anatomischen Achsstellung anzupassen.

\section{Präoperative Planung}

Mit einer guten Funktion des Kniegelenks ist zu rechnen, wenn der Oberflächenersatz sowohl von der Lastlinie als auch der Kniebasislinie als auch der Rotationsstellung her korrekt eingebracht wurde. Darüber hinaus bestimmt die Höhe der Resektionsebene des femoralen und tibialen Anteils die Gelenkspannung (Abb. 2).

Die präoperative subtile Planung stellt hier eine Conditio sine qua non dar. Die dreidimensionale Darstellung, basierend auf computertomographischen Bildern, bietet die optimale Voraussetzung, einen artifiziellen Oberflächenersatz unter Berücksichtigung der Gesamtfunktion des Beins optimal einzusetzen. Schwere Fehlstellungen, nicht nur im Bereich der Lastlinie, sondern auch bezüglich der Rotation, können erkannt und korrigiert werden. Ebenso er- laubt die Größendarstellung, die anders als das konventionelle Röntgenbild keine Größenverzerrung erfährt, die passgenaue Größenwahl (Abb. 3).

\section{Einsatz von Roboter- und Navigationssystemen}

Vor dem Hintergrund dieser hoch präzisen Planung, die heute nahezu Standard für jedes künstliches Gelenk sein sollte, erlaubt allein der Einsatz eines Roboter- bzw. Navigationssystems eine qualitätsverlustfreie Umsetzung der präoperativen Planung. Die individuellen Verhältnisse im Bereich der unteren Gliedmaßen, die vor allen Dingen im Bereich der Weichteile, besonders im Bereich der Hüfte, die korrekte Orientierung am Skelett erheblich erschweren, erlauben kein absolut präzises Wiederauffinden der präoperativ festgelegten Achse. Die Orientierung am Markraum bietet hier Annäherung, jedoch keine absolute Präzision. Durch den Einsatz der neuen Technologien kann dieser Nachteil ausgeglichen werden.

(c) Springer-Verlag 2003

Dr. Alexander Olk

Abteilung für Unfallchirurgie, Chirurgische Klinik mit Poliklinik,

Friedrich-Alexander-Universität Erlangen-

Nürnberg,

Krankenhausstraße 12,91054 Erlangen,

E-Mail: AlexOlk@aol.com,

Tel.: 09131-8533272, Fax: 09131-8533300 


\section{A. Olk ·.F.Hennig}

\section{Navigation and robotics in total knee arthroplasty. Current status}

\section{Abstract}

The wide application of robotics and electronics in the manufacture of virtually everything we use in everyday life leads us to ask whether it would not benefit our patients if these technologies were also applied in the setting of operative interventions. Such applications should certainly not be seen as attempts to replace the surgeon, but should be strictly restricted to precisely defined steps in the operations concerned and can thus be seen as an aid to an experienced surgeon. Following the successful introduction of robot-assisted navigation systems and surgery in the mid-1990s, these procedures and systems are now being applied more and more frequently in knee replacement operations.

\section{Keywords}

Robotics $\cdot$ Navigation $\cdot$ Total knee arthroplasty

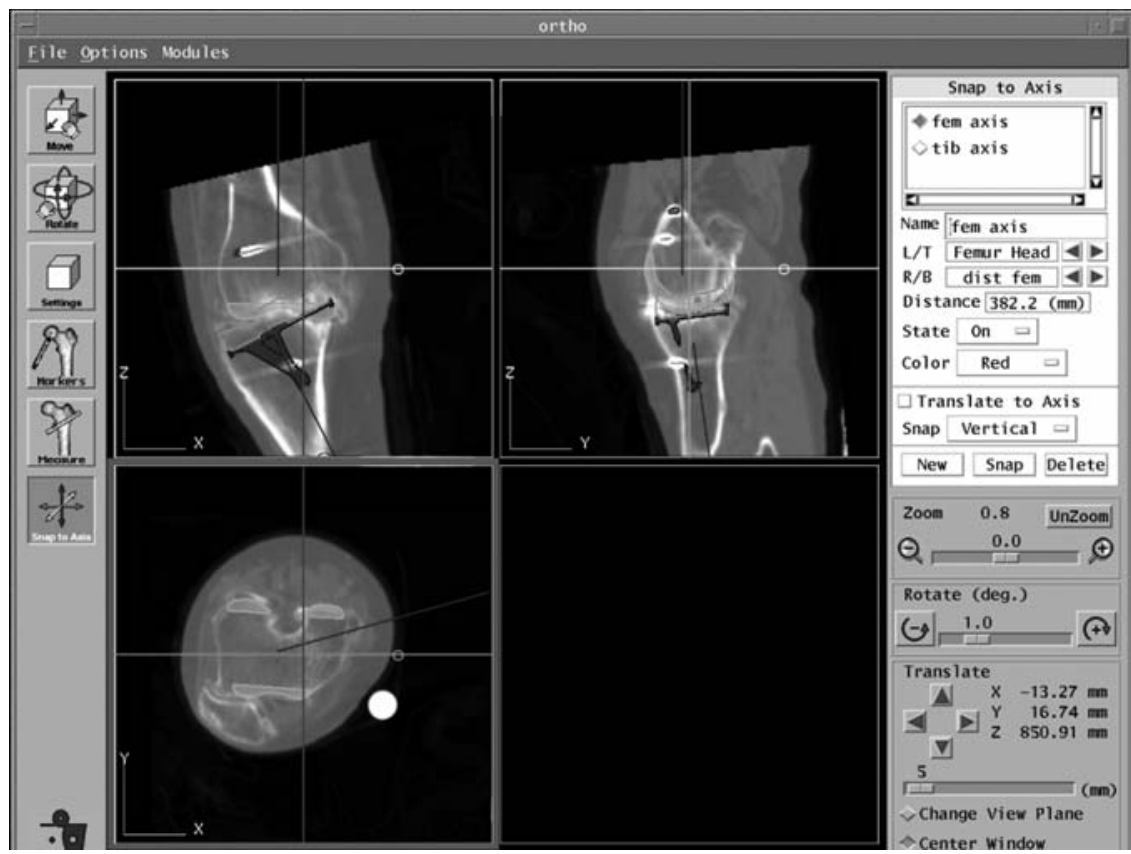

Abb. $2 \triangle$ Implantatwahl und -positionierung

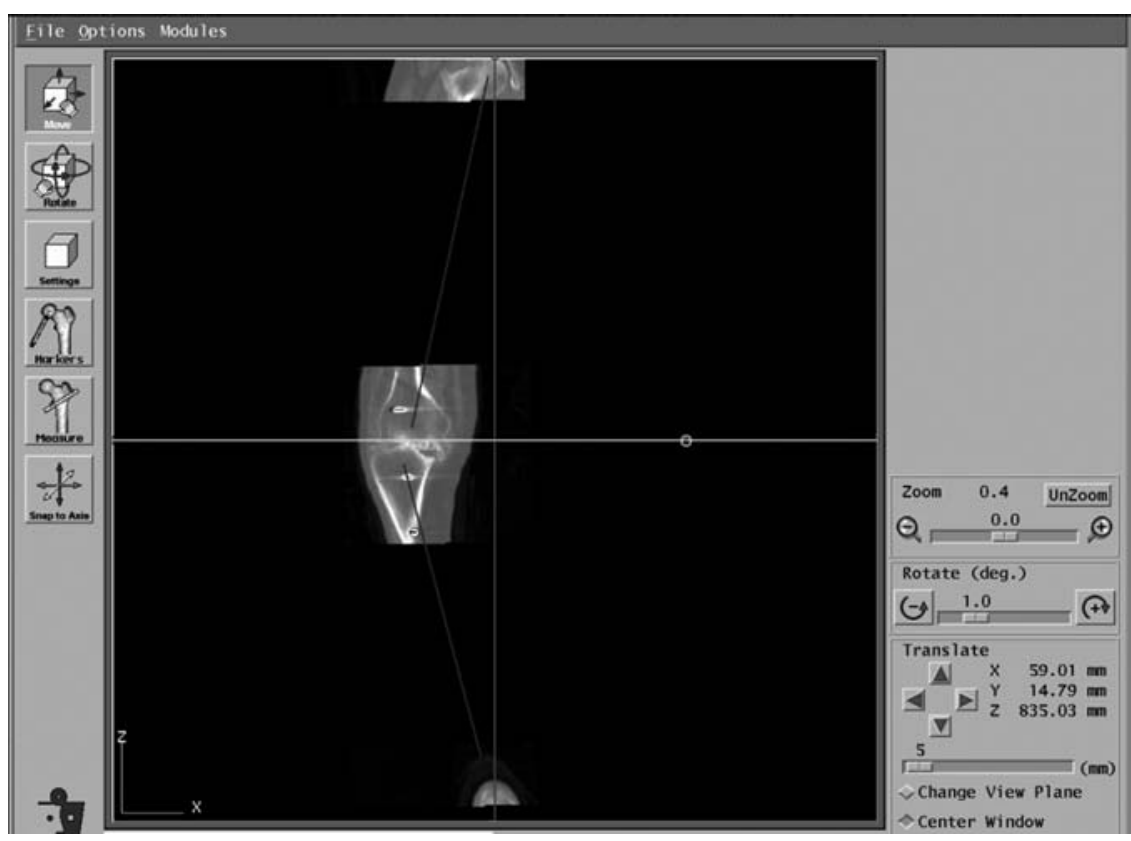

Abb. $1 \Delta$ Präoperative Achsbestimmung an der Planungsstation Orthodoc, ISS

\section{Ergebnisse nach Einsatz von Robotersystemen}

Die weltweit ersten robotergesteuerten Operationen am Kniegelenk wurden an der BG-Unfallklinik Frankfurt (Prof. Dr. M. Börner) und an der Abteilung für Unfallchirurgie, Friedrich-Alexander-Universität Erlangen-Nürnberg (Prof. Dr. F.
F. Hennig), im Februar 2000 durchgeführt. Seitdem wurden an der Abteilung für Unfallchirurgie, Friedrich-Alexander-Universität Erlangen-Nürnberg, 84 erfolgreiche Operationen mit Hilfe dieser Technik durchgeführt (Stand Februar 2001). Der operative Ablauf und die Integration der Robotertechnologie gestalteten sich weitgehend problemlos, die Operationszeit liegt nur unwesentlich über der der konventionellen Operationstechnik, der Blutverlust ist vergleichbar.

Die Positionsergebnisse des Implantats, kontrolliert durch postoperative Becken-Bein-Aufnahmen, waren in allen Fällen absolut achsgerecht, die Passgenauigkeit der gefrästen Oberfläche ideal. In der bisher kurzen Nachbeobachtungszeit wurden 2 Arthrofibrosen beobachtet, die zu einer Arthroskopie mit Arthrolyse führten. Möglicherweise ist die Ursache in den feinen knöchernen Fräspartikeln, welche in das Gewebe eindringen können, zu sehen. Aufgrund dieser Beobachtung setzen wir heute generell eine Jetlavage ein und nehmen eine intensive Reinigung des Operationssitus vor. Eine oberflächliche sowie eine tiefe Wundheilungsstörung sind als weitere Komplikationen zu nennen.

Die derzeit noch erforderliche etwa 20-minütige präoperative Pinmarkierungsoperation wird an unserer Klinik in der Regel in einer Spinalanästhesie 
Posttraumatische Gonarthrose

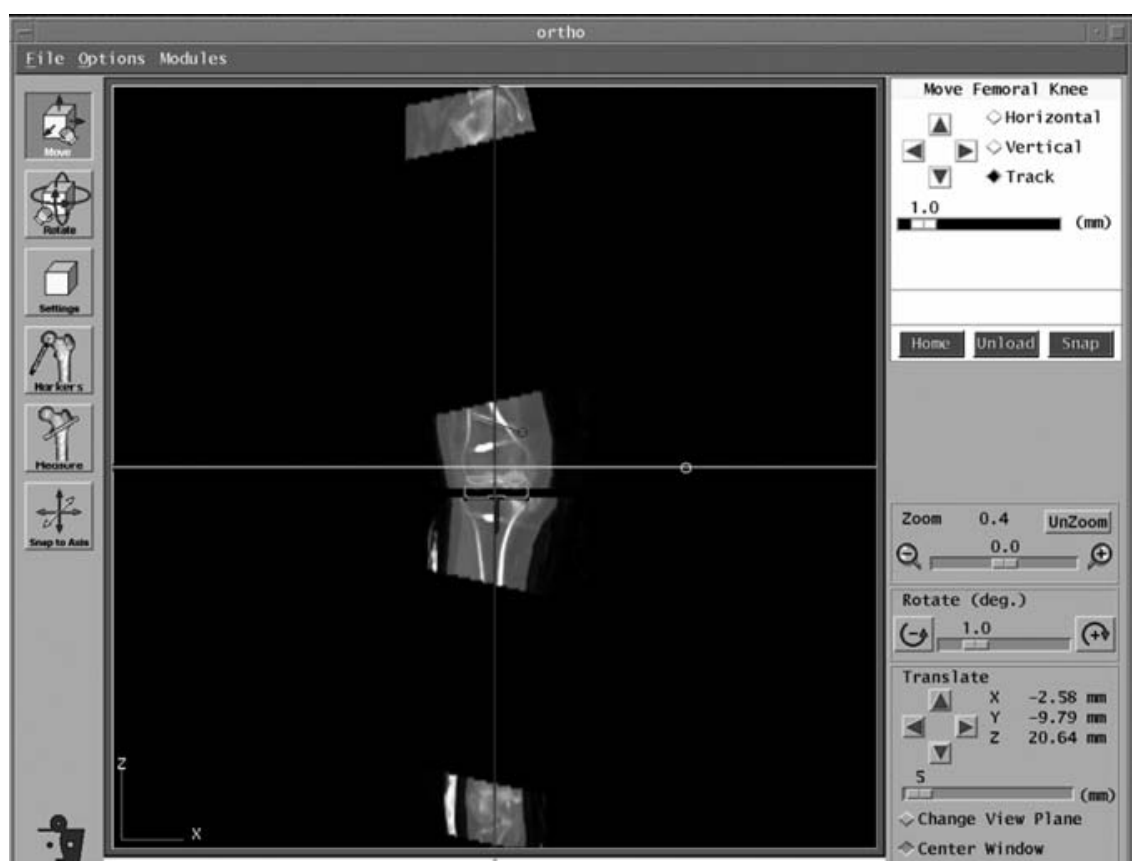

Abb. $3 \Delta$ Virtuelles postoperatives Ergebnis

durchgeführt. Der Patient wird dann umgehend in das CT verbracht, um noch die positiven Effekte der Spinalanästhesie zu nutzen. Nach durchgeführter CTUntersuchung schließt sich umgehend der operative Eingriff des alloarthroplastischen Gelenkersatzes unter Vollnarkose an.

Neben dem röntgenologisch nachgewiesenen, achsgerechten und passgenauen Sitz des Implantats haben wir die Fräsgenauigkeit des Roboters in Kooperation mit dem Institut für Physikalische Medizin, Universität Erlangen-Nürnberg, in vitro getestet. Es konnten hierbei sehr gute Ergebnisse erzielt werden. Die Abweichung der Gesamttechnologie unter Einschluss des CT, des Operateurs und des Roboters lag für alle gemessenen Werte weit unter dem mm-Bereich (Abb. 4). Die mechanische Oberflächenbearbeitung im Rahmen des elektronisch gesteuerten Fräsvorgangs bei ständiger Spülung zeigte in der feingeweblichen Aufarbeitung keinerlei Hitzeschädigung der trabekulären Struktur des Knochens sowie keine Zeichen einer Mikrofraktur.

\section{Resümee}

Insgesamt lassen sich die Vor- und Nachteile des Systems wie folgt darstellen:

Abb. $4 \Delta$ Wiederholungsgenauigkeit des Robodoc-System
Hier muss jedoch die Bedeutung des Eingriffs vor dem Hintergrund gesehen werden, dass die Strahlenbelastung durch ein Planungs-CT mit einer modernen Technologie heute bei durchschnittlich 2,5 mSv liegt und damit in der Größenordnung der natürlichen jährlichen Strahlenbelastung in unserer Region.

Der erforderliche erhebliche Schulungsaufwand des Operationsteams und der finanzielle Aufwand rechnen sich nur, wenn die Technologie hochfrequent zum Einsatz kommt.

\section{Navigationssysteme}

Die Navigationssysteme finden unter gleicher Zielsetzung ihren Einsatz. Es lassen sich grundsätzlich 2 verschiedene Grundprinzipien bezüglich der Registrierung unterscheiden:

\section{CT-basierte Navigationssysteme 2. CT-freie Navigationssysteme}

1. Vorteile

- optimale dreidimensionale präoperative Planung

- korrekte Ausführung des Plans

- exakte Fräsung der Oberfläche mit einer erhaltenen und intakten Knochenstruktur

2. Nachteile

- erforderliche zusätzliche Pinmarkierungsoperation,

- geringfügig erhöhte Strahlenbelastung durch das erforderliche CT.
Eine weitere interessante Neuerung beruht darauf, nicht den Operateur im Rahmen der einzelnen operativen Handgriffe navigiert zu führen, sondern eine kleinste Fräsung navigiert so zu positionieren, dass sie im Sinn eines „Miniroboters" den Knochenbearbeitungsvorgang von einer Plattform aus durchführt. Der große Vorteil der Navigationssysteme beruht darauf, dass eine präoperative Pinmarkierung in keinem Fall erforderlich ist. Die Objekterkennung erfolgt intraoperativ anhand des Aufsu-

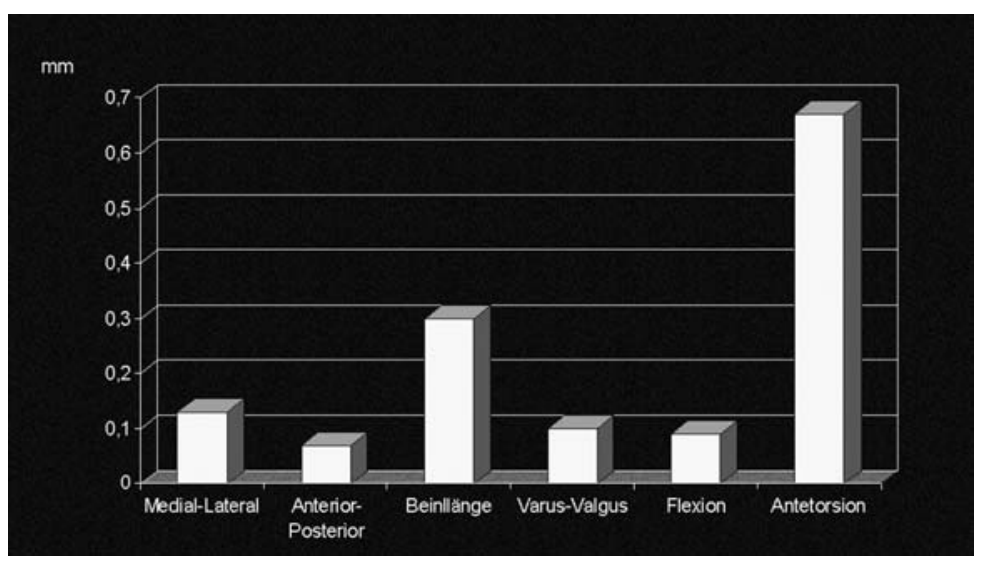


chens mehrerer anatomischer Landmarken am distalen Femur und der proximalen Tibia, Festlegung des Drehzentrums des Hüftkopfs und des Mittelpunkts des Sprunggelenks.

Bei den CT-gesteuerten Navigationssystemen ist vergleichbar mit der Robotik eine umfangreiche präoperative Planung möglich, bei den CT-freien Systemen erfolgt die Planung durch intraoperative Kommunikation zwischen Operateur und Elektronik. Das Einbinden des Operateurs in den direkten Umsetzungsvorgang hat zum einen den großen Vorteil, dass jeder Schritt operateurkontrolliert durchgeführt wird, bringt aber andererseits durch die Zwischenschaltung des Faktors Mensch in die elektronischmechanische Kopplung einen gewissen Stressfaktor mit sich. Darüber hinaus erfolgt die Oberflächenbearbeitung in aller Regel durch eine oszillierende Säge, die ihrerseits eine Bearbeitungsungenauigkeit mit sich bringt und darüber hinaus oberflächentraumatisierend arbeitet.

\section{Diskussion}

Beim Vergleich zwischen roboterassistierten und navigierten Systemen kann zum jetzigen Zeitpunkt keine Wertung getroffen werden (Tabelle 1). Beide Technologien zielen auf eine Verbesserung der Implantatpräzision ab. Sie bieten die Chance standardisierter, reproduzierbarer Implantatpositionen und reduzieren damit deutlich die Anzahl der unbekannten Variablen im Rahmen des Operationsablaufs. Nur durch diesen Vorgang ist es möglich, Operationsergebnisse und Langzeitergebnisse vor gesicherten standardisierten Daten zu überprüfen und Rückschlüsse bezüglich Implantatlage und Implantatdesign langfristig zu treffen.

Der Qualitätsstandard beider Technologien erlaubt ihren Einsatz am Menschen in der Hand des erfahrenen Operateurs bei fundierter Schulung des ärztlichen und pflegerischen Personals. Beide Technologien, in Zukunft mit hoher Wahrscheinlichkeit synergistisch, lassen einen erheblichen Fortschritt in der Endoprothetik erwarten.

\section{Tabelle 1 \\ Vergleich Robotik und Navigation}

\begin{tabular}{lll} 
Parameter & Roboter & Navigation \\
\hline Lage des Implantats & ++ & + \\
Passgenauigkeit & ++ & $(+)$ \\
Operationstrauma & 0 & 0 \\
Operationszeit & 0 & 0 \\
Flexibilität & - & ++ \\
Kosten & - & -
\end{tabular}

Haben wir bisher unsere Implantate im Bereich des Hüftgelenks bezogen auf einen relativ kleinen Skelettabschnitt, im Bereich des Kniegelenks wenigstens bezogen auf die gesamte untere Extremität implantiert, sollte in Zukunft unter Ausschöpfung der oben genannten Methoden und ihrer zu erwartenden zukünftigen Entwicklung eine lastgerechte Implantation eines künstlichen Gelenks unter Berücksichtigung des gesamten Kraftflusses des individuellen menschlichen Körpers, unter Berücksichtigung dynamischer Komponenten der Ganganalyse und der virtuellen präoperativen Funktionsanalyse möglich sein. Marketinggedanken haben bei der Beurteilung dieser modernen Technologie genauso wenig eine Berechtigung wie traditionalistisches Beharren auf lieb gewonnenen Operationspraktiken. Emotionsfreie, kritische, fachkundige Beurteilung im Interesse unserer Patienten ist gefragt.

\section{Weiterführende Literatur}

1. Archibeck MJ, White RE Jr (2001) What's new in adult reconstructive knee surgery.J Bone Joint Surg Am 83-A: 1444-1450

2. Blomer W (2000) Knee endoprostheses: problems and technological developments from the manufacturer's point of view. Orthopäde 29: 688-696

3. Brandt G, Radermacher K, Zimolong A, Rau G, Merloz P, Klos TV, Robb J, Staudte HW (2000) CRIGOS: development of a compact robot for image-guided orthopedic surgery. Orthopäde 29: 645-649

4. Bren $L$ (2000) Robots help surgeons perform more precise surgery. FDA Consum 34: 12-13

5. Davies BL, Harris SJ, Lin WJ, Hibberd RD, Middleton R, Cobb JC (1997) Active compliance in robotic surgery - the use of force control as a dynamic constraint. Proc Inst Mech Eng $[\mathrm{H}]$ 211: 285-292
6. Delp SL, Stulberg SD, Davies B, Picard F, Leitner F (1998) Computer assisted knee replacement. Clin Orthop 354: 49-56

7. DiGioia AM 3rd, Jaramaz B (1999) Computerassisted tools and interventional technologies. Lancet [Suppl] 354: SIV46

8. Englmeier KH, Haubner M, Krapichler C (1998) Principles and current possibilities of virtual scenarios for surgery planning. Langenbecks Arch Chir Suppl Kongressbd 115: 93-100

9. Gebhard F, Arand M, Fleiter T, Hebecker A, Heeckt $P$, Hesser J, Messmer P, Hufner T, Visarius H, Regazzoni P, KinzI L (2001) Computer assisted surgery, 2001 development and prospects. Results of a congress at Reisensburg Castle, 23-24 November 2000. Orthopäde 30: 666671

10. Gosse F, Brack C, Gotte H, Roth M, Ruhmann O, Schweikard A, Vahldiek M (1997) Roboterunterstützung in der Knieendoprothetik. Orthopäde 26: 258-266

11. Kerschbaumer $F(2000)$ „Numerische Bildgebung - Operationsplanung - Simulation - Navigation - Robotik"? Orthopäde 29: 597-598

12. Kinzl L (2000) Computer-assisted surgery. Unfallchirurg 103: 611

13. Lavallee S, Bainville E, Bricault I (2000) An overview of computer-integrated surgery and therapy. Crit Rev Diagn Imaging 41: 157-236

14. Martelli M, Marcacci M, Nofrini L, La Palombara F, Malvisi A, lacono F, Vendruscolo P, Pierantoni M (2000) Computer- and robot-assisted total knee replacement: analysis of a new surgical procedure. Ann Biomed Eng 28: 1146-1153

15. Schiffers N, Schkommodau E, Portheine F, Radermacher K, Staudte HW (2000) Planning and performance of orthopedic surgery with the help of individual templates. Orthopäde 29: 636-640

16. Schlondorff G (1998) Computer-assisted surgery: historical remarks. Comput Aided Surg 3: 150-152

17. Simon DA, Lavallee $S$ (1998) Medical imaging and registration in computer assisted surgery. Clin Orthop 354: 17-27

18. Thoma W, Schreiber S, Hovy L (2000) Computer-assisted implant positioning in knee endoprosthetics. Kinematic analysis for optimization of surgical technique. Orthopäde 29: 614626

19. Urban V, Wapler M, Neugebauer J, Hiller A, Stallkamp J, Weisener T (1998) Robot-assisted surgery system with kinesthetic feedback. Comput Aided Surg 3: 205-209

20. Wirth CJ (2000) The right way to the future of knee endoprostheses. Orthopäde 29: 685 